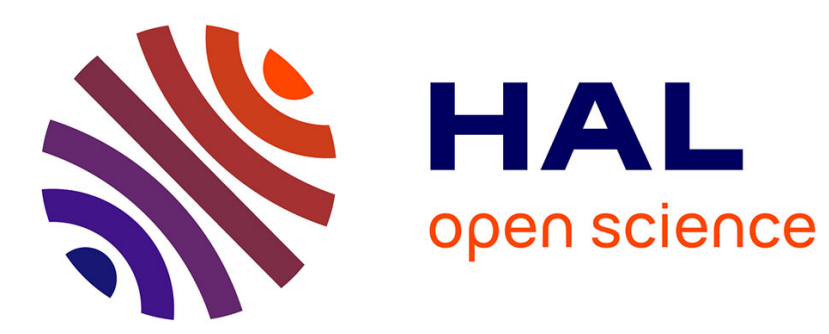

\title{
Sensor fault estimation filter design for discrete-time linear time-varying systems
}

Zhenhua Wang, Mickael Rodrigues, Didier Theilliol, Yi Shen

\section{To cite this version:}

Zhenhua Wang, Mickael Rodrigues, Didier Theilliol, Yi Shen. Sensor fault estimation filter design for discrete-time linear time-varying systems. Acta Automatica Sinica, 2014, 40 (10), pp.2364-2369. 10.1016/S1874-1029(14)60365-7 . hal-00989944

\section{HAL Id: hal-00989944 https://hal.science/hal-00989944}

Submitted on 12 May 2014

HAL is a multi-disciplinary open access archive for the deposit and dissemination of scientific research documents, whether they are published or not. The documents may come from teaching and research institutions in France or abroad, or from public or private research centers.
L'archive ouverte pluridisciplinaire HAL, est destinée au dépôt et à la diffusion de documents scientifiques de niveau recherche, publiés ou non, émanant des établissements d'enseignement et de recherche français ou étrangers, des laboratoires publics ou privés. 


\section{Sensor Fault Estimation Filter Design for Discrete-Time Linear Time-Varying Systems}

\author{
WANG Zhen-Hua ${ }^{1}$ RODRIGUES Mickael ${ }^{2}$ \\ THEILLIOL Didier ${ }^{3} \quad$ SHEN Yi $^{1}$
}

Abstract This paper proposes a sensor fault diagnosis method for a class of discrete-time linear time-varying (LTV) systems. In this paper, the considered system is firstly formulated as a descriptor system representation by considering the sensor faults as auxiliary state variables. Based on the descriptor system model, a fault estimation filter which can simultaneously estimate the state and the sensor fault magnitudes is designed via a minimumvariance principle. Then, a fault diagnosis scheme is presented by using a bank of the proposed fault estimation filters. The novelty of this paper lies in developing a sensor fault diagnosis method for discrete LTV systems without any assumption on the dynamic of fault. Another advantage of the proposed method is its ability to detect, isolate and estimate sensor faults in the presence of process noise and measurement noise. Simulation results are given to illustrate the effectiveness of the proposed method.

Key words Fault estimation, linear time-varying (LTV) systems, sensor faults, descriptor system, minimum-variance filter

Citation Wang Zhen-Hua, Rodrigues Mickael, Theilliol Didier, Shen Yi. Sensor Fault Estimation Filter Design for DiscreteTime Linear Time-Varying Systems. Acta Automatica Sinica, 2014, 40(1): 1-3

DOI $10.3724 /$ SP.J.1004.2012.xxxxx

With the growing complexity of modern engineering systems and ever increasing demand for safety and reliability, fault diagnosis techniques have received considerable attention during the past decades. Fruitful results can be found in several excellent monographs ${ }^{[1-3]}$, survey papers ${ }^{[4,5]}$ and the references therein. In the literature, model-based fault detection and isolation (FDI) approaches have been most widely considered. The fundamental idea behind FDI is to generate an alarm when the fault occurs, and then to determine the location of fault. However, the fault magnitude cannot be provided by the FDI methods.

Parallel to FDI, the fault estimation methods for dynamic systems have also been investigated by a number of scholars. Reference [6] proposed an actuator fault estimation method based on adaptive observer. Adaptive observer-based fault estimation methods for nonlinear systems were studied in $[7,8]$. In [9], online learning methodology was used to deal with fault estimation problem for nonlinear dynamic systems. Most recently, proportionalintegral observer has been used to achieve fault estimation in descriptor systems ${ }^{[10,11]}$. However, these aforementioned methods are only studied on fault estimation for continuous-time systems. On the contrary, little attention has been paid to fault estimation in discrete-time systems. Reference [12] considered fault estimation of actuator faults

Manuscript received Month Date, Year; accepted Month Date, Year Supported by National Natural Science Foundation of China (61273162)

Recommended by Associate Editor BIAN Wei

1. School of Astronautics, Harbin Institute of Technology, Harbin 150001, P. R. China 2. Automatic and Process Control Laboratory, University of Lyon, Lyon, F-69003, France; Université Lyon 1,CNRS UMR 5007, Villeurbanne, F-69622, France 3. Centre de Recherche en Automatique de Nancy, Université de Lorraine, CNRS UMR 7039, F-54506 Vandoeuvre-les-Nancy, France for linear multi-input-multi-output systems. In [13], a fault estimation method based on proportional integral observer was proposed for a class of discrete-time nonlinear systems. However, these methods mainly focus on time-invariant systems with actuator faults. In [14], the authors proposed a fault diagnosis method for discrete-time descriptor linear parameter-varying systems. However, the method in [14] requires the fault to be constant or slow varying, which is a restrictive condition. [15] proposed an $H_{\infty}$ fault estimation method for linear time-varying (LTV) systems based on Krein space approach. However, the control input is not considered in [15].

Recently, references $[16,17]$ have proposed a descriptor system approach to deal with sensor fault estimation problem. The basic idea behind this method is to construct an augmented descriptor system so that the simultaneous state and fault estimation problem is transformed as the state estimation of the augmented descriptor plant. This methodology provides a novel solution for sensor fault estimation. However, $[16,17]$ only studied sensor fault estimation for continuous-time systems. Moreover, process noise and measurement noise are not considered in these works. In contrast to continuous-time systems, there are few results on sensor fault estimation for discrete-time systems in the literature. Most recently, the authors in [18] have proposed a sensor fault detection, isolation and estimation method for discrete-time Linear Parameter-Varying (LPV) systems. The method in [18] assumes the dynamic of fault can be described by a known model, which is difficult to be determined previously. This assumption restricts the applicable scope of the method in [18]. Moreover, if the dynamic model of fault is not properly chosen, it will leads to undesirable fault diagnosis results. In [19], a sensor fault estimation method for discrete-time systems is proposed by using descriptor Kalman filter. However, [19] only concerns the Linear Time-Invariant (LTI) systems. To the best of our knowledge, sensor fault estimation for discrete-time LTV systems has not been fully investigated, which motivates the presented work.

This paper proposes a sensor fault diagnosis approach for LTV systems. Firstly, a fault estimation filter which can can simultaneously estimate the state and the sensor fault magnitudes is designed by using the descriptor system technique. Then, a fault diagnosis scheme is presented by using a bank of the proposed fault estimation filters. The main contribution lies in two aspects. First, A new fault diagnosis method which is able to detect, isolate and estimate sensor faults in discrete LTV systems. Compared with the existing result in [19], the proposed method is appliable to LTV systems, which is more challenge to deal with than LTI systems. In comparison with the method in [18], the proposed approach does not make any assumption on the dynamic of fault. As a result, the latter has a broader applicable scope and can be used to deal with time-varying faults. Moreover, both process noise and measurement noise are considered in this paper, which makes the presented approach practical for real systems.

\section{Problem formulation}

Consider the following discrete-time LTV system

$$
\left\{\begin{array}{l}
x_{k+1}=A_{k} x_{k}+B_{k} u_{k}+D_{k} w_{k} \\
y_{k}=C_{k} x_{k}+F_{k} f_{k}+v_{k}
\end{array}\right.
$$

where $x_{k} \in \mathbb{R}^{n}, u_{k} \in \mathbb{R}^{p}, y_{k} \in \mathbb{R}^{m}, w_{k} \in \mathbb{R}^{l}$ and $v_{k} \in \mathbb{R}^{m}$ are the state, control input, output, process noise, and measurement noise vectors, respectively. $A_{k}$, 
$B_{k}, C_{k}$ and $D_{k}$ are known matrices of appropriate dimensions. It is assumed that $w_{k}$ and $v_{k}$ are uncorrelated white noises with covariance matrices $Q=\mathbb{E}\left[w_{k} w_{k}^{T}\right] \geq 0$ and $R=\mathbb{E}\left[v_{k} v_{k}^{T}\right]>0$, respectively. The initial state $x_{0}$ is of mean $\bar{x}_{0}$ and covariance $P_{0}$ and is independent of $w_{k}$ and $v_{k} . F_{k} \in \mathbb{R}^{m \times q}$ represents the sensor fault distribution matrix, and the unknown signal $f_{k} \in \mathbb{R}^{q}$ denotes the effect of the sensor faults. Without loss of generality, it is assumed that matrix $F_{k}$ satisfies

$$
\operatorname{rank}\left(F_{k}\right)=q, q<m
$$

which implies the the number of faults is less than that of measurements. This assumption is reasonable since the probability for faults to occur at all sensors is very small in practice. It should be noted that the fault distribution matrix $F_{k}$ is unknown since different fault modes may occur. Therefore, it is reasonable to assume that $F_{k}$ belongs to a given set, i.e.

$$
F_{k} \in \mathcal{F}_{k} \triangleq\left\{F_{k}^{1}, F_{k}^{2}, \cdots, F_{k}^{M}\right\}
$$

Herein, $M$ is the number of possible fault modes.

The main purpose of this paper is to determine the current fault mode $F_{k}$ and to obtain the estimate for the fault magnitude $f_{k}$. To this end, this paper proposes a filter synthesis approach to achieve fault estimation for a specific fault mode, and then presents a fault diagnosis scheme based on a bank of dedicated filters.

\section{Fault estimation filter design}

In this section, a fault estimation filter is designed for a specific fault mode. In this section, the fault distribution matrix is denoted by $F_{k}$. However, this representation is only used for the convenience of statement because the fault estimation filter synthesis is an essential basis for the fault diagnosis scheme which will be presented in the next section.

To estimate sensor fault, an augmented state vector is defined as

$$
\bar{x}_{k}=\left[\begin{array}{l}
x_{k} \\
f_{k}
\end{array}\right]
$$

Then, the system (1) with sensor fault can be written as the following descriptor system

$$
\left\{\begin{array}{l}
E \bar{x}_{k+1}=\bar{A}_{k} \bar{x}_{k}+\bar{B}_{k} u_{k}+\bar{D}_{k} w_{k} \\
y_{k}=\bar{C}_{k} \bar{x}_{k}+v_{k}
\end{array}\right.
$$

where

$$
\begin{gathered}
E=\left[\begin{array}{cc}
I_{n} & 0 \\
0 & 0
\end{array}\right], \bar{A}_{k}=\left[\begin{array}{cc}
A_{k} & 0 \\
0 & 0
\end{array}\right], \bar{B}_{k}=\left[\begin{array}{c}
B_{k} \\
0
\end{array}\right] \\
\bar{D}_{k}=\left[\begin{array}{c}
D_{k} \\
0
\end{array}\right], \bar{C}_{k}=\left[\begin{array}{ll}
C_{k} & F_{k}
\end{array}\right]
\end{gathered}
$$

If a state estimator is designed for descriptor system (5), then the state $x_{k}$ and sensor fault $f_{k}$ in system (1) can be estimated simultaneously. In other words, by constructing descriptor system (5), simultaneous state and fault estimation for system (1) is converted into a state estimation problem of descriptor system (5).

Motivated by the observer proposed in [20], the following filter is constructed for descriptor system (5)

$$
\hat{\bar{x}}_{k+1}=T_{k} \bar{A}_{k} \hat{\bar{x}}_{k}+T_{k} \bar{B}_{k} u_{k}+L_{k}\left(y_{k}-\bar{C}_{k} \hat{\bar{x}}_{k}\right)+N_{k} y_{k+1}
$$

where $\hat{\bar{x}}_{k} \in \mathbb{R}^{n+q}$ denotes the estimation of the descriptor state $\bar{x}_{k}, T_{k} \in \mathbb{R}^{(n+q) \times(n+q)}, N_{k} \in \mathbb{R}^{(n+q) \times m}$ and $L_{k} \in$ $\mathbb{R}^{(n+q) \times m}$ are matrices to be designed.

In filter (7), matrices $T_{k}$ and $N_{k}$ are designed to satisfy the following equation

$$
T_{k} E+N_{k} \bar{C}_{k+1}=I_{n+q}
$$

To proceed, we introduce the following Lemma which will be used in the sequel.

Lemma 1. For given matrices $\mathcal{B}$ and $\mathcal{Y}$, there exists a matrix $\mathcal{X}$ that satisfy $\mathcal{X} \mathcal{B}=\mathcal{Y}$ if and only if

$$
\operatorname{rank}\left[\begin{array}{c}
\mathcal{B} \\
\mathcal{Y}
\end{array}\right]=\operatorname{rank}(\mathcal{Y})
$$

Moreover, a general solution to $\mathcal{X B}=\mathcal{Y}$ is given by

$$
\mathcal{X}=\mathcal{Y} \mathcal{B}^{\dagger}+\mathcal{S}\left(I-\mathcal{B B}^{\dagger}\right)
$$

where $\mathcal{B}^{\dagger}$ denotes the pseudo-inverse of $\mathcal{B}$ and $\mathcal{S}$ is an arbitrary matrix.

Proof. Lemma 1 is a straightforward result of the Theorem of Penrose ${ }^{[21]}$.

Since $\operatorname{rank}\left(F_{k}\right)=q$, it is easy to show that

$$
\operatorname{rank}\left[\begin{array}{c}
E \\
\bar{C}_{k+1}
\end{array}\right]=n+q
$$

According to Lemma 1, there exist a matrix $\left[\begin{array}{ll}T_{k} & N_{k}\end{array}\right]$ satisfying

$$
\left[\begin{array}{ll}
T_{k} & N_{k}
\end{array}\right]\left[\begin{array}{c}
E \\
\bar{C}_{k+1}
\end{array}\right]=I_{n+q}
$$

i.e. there exist two matrices $T_{k}$ and $N_{k}$ such that equation (8) holds.

By using Lemma 1 , matrices $T_{k}$ and $N_{k}$ can be determined by

$$
\begin{aligned}
& T_{k}=\Theta^{\dagger} \alpha_{1}+S\left(I_{n+q+m}-\Theta \Theta^{\dagger}\right) \alpha_{1} \\
& N_{k}=\Theta^{\dagger} \alpha_{2}+S\left(I_{n+q+m}-\Theta^{\dagger}\right) \alpha_{2}
\end{aligned}
$$

where $S \in \mathbb{R}^{(n+q) \times(n+q+m)}$ is an arbitrary matrix providing degrees of freedom, matrices $\Theta \in \mathbb{R}^{(n+q+m) \times(n+q)}, \alpha_{1} \in$ $\mathbb{R}^{(n+q+m) \times(n+q)}$ and $\alpha_{2} \in \mathbb{R}^{(n+q+m) \times m}$ are given by

$$
\Theta=\left[\begin{array}{c}
E \\
\bar{C}_{k+1}
\end{array}\right], \alpha_{1}=\left[\begin{array}{c}
I_{n+q} \\
0
\end{array}\right], \alpha_{2}=\left[\begin{array}{c}
0 \\
I_{m}
\end{array}\right]
$$

For the convenience of statement, the estimation error is denoted as

$$
e_{k}=\bar{x}_{k}-\hat{\bar{x}}_{k}
$$

and the error covariance matrix $P_{k}$ is defined as

$$
P_{k}=\mathbb{E}\left[e_{k} e_{k}^{T}\right]
$$

Now, the following Theorem is proposed to design matrix $L_{k}$ in filter (7) by minimizing the trace of the estimation error covariance matrix $P_{k+1}=\mathbb{E}\left[e_{k+1} e_{k+1}^{T}\right]$. 
Theorem 1. The gain matrix $L_{k}$ given by

$$
L_{k}=T_{k} \bar{A}_{k} P_{k} \bar{C}_{k}^{T}\left[\bar{C}_{k} P_{k} \bar{C}_{k}^{T}+R\right]^{-1}
$$

minimizes the trace of $P_{k+1}$. Moreover, the estimation error covariance matrix $P_{k}$ can be updated by

$$
\begin{gathered}
P_{k+1}=T_{k} \bar{A}_{k} P_{k}\left(T_{k} \bar{A}_{k}\right)^{T}-L_{k} \bar{C}_{k} P_{k}\left(T_{k} \bar{A}_{k}\right)^{T} \\
+T_{k} \bar{D}_{k} Q \bar{D}_{k}^{T} T_{k}^{T}+N_{k} R N_{k}^{T}
\end{gathered}
$$

Proof. Using (5) and (7), the error dynamic equation is obtained as follows

$$
\begin{aligned}
e_{k+1}= & \left(T_{k} E+N_{k} \bar{C}_{k+1}\right) \bar{x}_{k+1}-\hat{\bar{x}}_{k+1} \\
= & \left(T_{k} \bar{A}_{k}-L_{k} \bar{C}_{k}\right) e_{k}+T_{k} \bar{D}_{k} w_{k} \\
& -L_{k} v_{k}-N_{k} v_{k+1}
\end{aligned}
$$

From equation (20), it is easy to derive that

$$
\begin{aligned}
P_{k+1}= & \mathbb{E}\left[e_{k+1} e_{k+1}^{T}\right] \\
= & \left(T_{k} \bar{A}_{k}-L_{k} \bar{C}_{k}\right) P_{k}\left(T_{k} \bar{A}_{k}-L_{k} \bar{C}_{k}\right)^{T} \\
& \quad+T_{k} \bar{D}_{k} Q \bar{D}_{k}^{T} T_{k}^{T}+L_{k} R L_{k}^{T}+N_{k} R N_{k}^{T} \\
= & T_{k} \bar{A}_{k} P_{k}\left(T_{k} \bar{A}_{k}\right)^{T}+T_{k} \bar{D}_{k} Q \bar{D}_{k}^{T} T_{k}^{T}+N_{k} R N_{k}^{T} \\
& \quad-L_{k} \bar{C}_{k} P_{k}\left(T_{k} \bar{A}_{k}\right)^{T}-T_{k} \bar{A}_{k} P_{k} \bar{C}_{k}^{T} L_{k}^{T} \\
& +L_{k}\left(\bar{C}_{k} P_{k} \bar{C}_{k}^{T}+R\right) L_{k}^{T}
\end{aligned}
$$

Since $R$ is a positive definite matrix, $\bar{C}_{k} P_{k} \bar{C}_{k}^{T}+R$ is also positive definite. Consequently, there exists a nonsingular matrix $G_{k} \in \mathbb{R}^{m \times m}$ satisfying

$$
G_{k} G_{k}^{T}=\bar{C}_{k} P_{k} \bar{C}_{k}^{T}+R
$$

Substituting (22) into (21) yields

$$
\begin{aligned}
P_{k+1}= & T_{k} \bar{A}_{k} P_{k}\left(T_{k} \bar{A}_{k}\right)^{T}+T_{k} \bar{D}_{k} Q \bar{D}_{k}^{T} T_{k}^{T}+N_{k} R N_{k}^{T} \\
& -L_{k} \bar{C}_{k} P_{k}\left(T_{k} \bar{A}_{k}\right)^{T}-T_{k} \bar{A}_{k} P_{k} \bar{C}_{k}^{T} L_{k}^{T} \\
& +L_{k} G_{k} G_{k}^{T} L_{k}^{T}
\end{aligned}
$$

Letting

$$
H_{k}=T_{k} \bar{C}_{k} P_{k} \bar{C}_{k}^{T}\left(G_{k}\right)^{-1}
$$

and substituting (24) into (23) yields

$$
\begin{gathered}
P_{k+1}=T_{k} \bar{A}_{k} P_{k}\left(T_{k} \bar{A}_{k}\right)^{T}+T_{k} \bar{D}_{k} Q \bar{D}_{k}^{T} T_{k}^{T}+N_{k} R N_{k}^{T} \\
-L_{k} G_{k} P_{k} H_{k}^{T}-H_{k} G_{k}^{T} L_{k}^{T}+L_{k} G_{k} G_{k}^{T} L_{k}^{T} \\
=T_{k} \bar{A}_{k} P_{k}\left(T_{k} \bar{A}_{k}\right)^{T}+T_{k} \bar{D}_{k} Q \bar{D}_{k}^{T} T_{k}^{T}+N_{k} R N_{k}^{T} \\
\quad-L_{k} G_{k} P_{k} H_{k}^{T}-H_{k} G_{k}^{T} L_{k}^{T}+L_{k} G_{k} G_{k}^{T} L_{k}^{T} \\
\quad+H_{k} H_{k}^{T}-H_{k} H_{k}^{T} \\
=T_{k} \bar{A}_{k} P_{k}\left(T_{k} \bar{A}_{k}\right)^{T}+T_{k} \bar{D}_{k} Q \bar{D}_{k}^{T} T_{k}^{T}+N_{k} R N_{k}^{T} \\
\left(L_{k} G_{k}-H_{k}\right)\left(L_{k} G_{k}-H_{k}\right)^{T}-H_{k} H_{k}^{T}
\end{gathered}
$$

From (25), it is obvious that the trace of $P_{k+1}$ is minimized by letting

$$
L_{k} G_{k}-H_{k}=0
$$

Post-multiplying (26) by $G_{k}^{T}$, it comes

$$
L_{k}\left(\bar{C}_{k} P_{k} \bar{C}_{k}^{T}+R\right)-T_{k} \bar{A}_{k} P_{k} \bar{C}_{k}^{T}=0
$$

Solving equation (27) gives (18).

On the other hand, substituting (26) into (25) gives

$$
\begin{aligned}
P_{k+1}= & T_{k} \bar{A}_{k} P_{k}\left(T_{k} \bar{A}_{k}\right)^{T}-H_{k} H_{k}^{T} \\
& +T_{k} \bar{D}_{k} Q \bar{D}_{k}^{T} T_{k}^{T}+N_{k} R N_{k}^{T}
\end{aligned}
$$

Using (24) and (22), it can be derived that

$$
\begin{gathered}
P_{k+1}=T_{k} \bar{A}_{k} P_{k}\left(T_{k} \bar{A}_{k}\right)^{T}+T_{k} \bar{D}_{k} Q \bar{D}_{k}^{T} T_{k}^{T}+N_{k} R N_{k}^{T} \\
\quad-T_{k} \bar{A}_{k} P_{k} \bar{C}_{k}^{T}\left(G_{k} G_{k}^{T}\right)^{-1} \bar{C}_{k} P_{k}\left(T_{k} \bar{A}_{k}\right)^{T} \\
=T_{k} \bar{A}_{k} P_{k}\left(T_{k} \bar{A}_{k}\right)^{T}+T_{k} \bar{D}_{k} Q \bar{D}_{k}^{T} T_{k}^{T}+N_{k} R N_{k}^{T} \\
\quad-T_{k} \bar{A}_{k} P_{k} \bar{C}_{k}^{T}\left(\bar{C}_{k} P_{k} \bar{C}_{k}^{T}+R\right)^{-1} \bar{C}_{k} P_{k}\left(T_{k} \bar{A}_{k}\right)^{T}
\end{gathered}
$$

Substituting (18) into (29), we obtain (19).

Remark 1. Although the descriptor system approach has been used to deal with sensor fault estimation problem, most of the existing results focus on continuous-time systems $^{[16,17]}$. Compared to the existing works, the main contribution of this paper consists in two folds. First, a new sensor fault estimation method for discrete-time LTV systems is proposed. Second, a minimum-variance filter is designed to optimize the fault estimation performance in the presence of process noise and measurement noise.

\section{Fault diagnosis scheme}

In Section 2, a filter is designed to estimate the sensor faults associated with fault distribution matrix $F_{k}$. However, different fault mode leads to different fault distribution matrix $F_{k}$. Therefore, it is also desirable to find out which sensors are faulty when faults have occurred. In this section, we present a fault diagnosis strategy which is similar to the well-known Dedicated Observer Scheme (DOS). Fig. 1 illustrates the basic structure of the proposed fault diagnosis scheme.

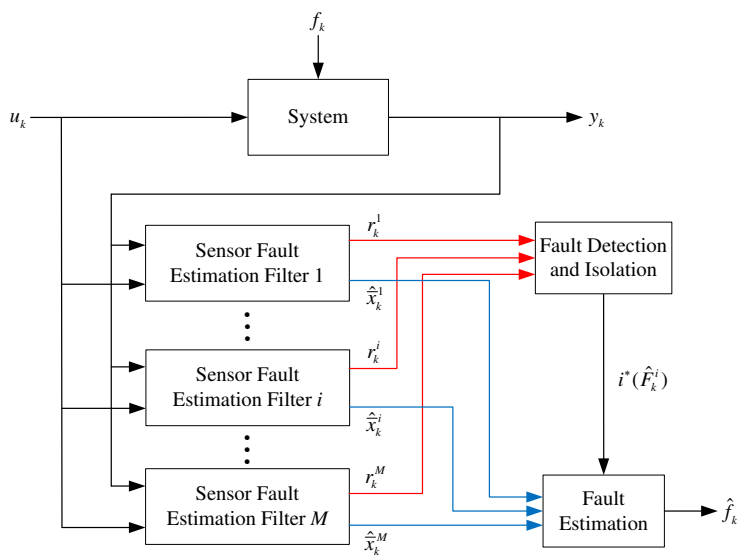

Figure 1 Basic structure of the fault diagnosis scheme

The detail principle of the proposed fault diagnosis scheme is stated in the following.

Since there are $M$ possible fault modes, all possible faulty models are given as follows

$$
\left\{\begin{array}{l}
x_{k+1}=A_{k} x_{k}+B_{k} u_{k}+D_{k} w_{k} \\
y_{k}=C_{k} x_{k}+F_{k}^{i} f_{k}+v_{k}
\end{array}, i=1, \cdots, M\right.
$$

Then, the faulty models (30) can be formulated as the following descriptor representation

$$
\left\{\begin{array}{l}
E \bar{x}_{k+1}=\bar{A}_{k} \bar{x}_{k}+\bar{B}_{k} u_{k}+\bar{D}_{k} w_{k}, i=1, \cdots, M \\
y_{k}=\bar{C}_{k}^{i} \bar{x}_{k}+v_{k}
\end{array}\right.
$$


where

$$
\begin{gathered}
E=\left[\begin{array}{cc}
I_{n} & 0 \\
0 & 0
\end{array}\right], \bar{A}_{k}=\left[\begin{array}{cc}
A_{k} & 0 \\
0 & 0
\end{array}\right], \bar{B}_{k}=\left[\begin{array}{c}
B_{k} \\
0
\end{array}\right] \\
\bar{D}_{k}=\left[\begin{array}{c}
D_{k} \\
0
\end{array}\right], \bar{C}_{k}^{i}=\left[\begin{array}{ll}
C_{k} & F_{k}^{i}
\end{array}\right]
\end{gathered}
$$

Then, a bank of sensor fault estimation filters are constructed as follows

$$
\left\{\begin{array}{l}
\hat{\bar{x}}_{k+1}^{i}=T_{k}^{i} \bar{A}_{k} \hat{\bar{x}}_{k}+T_{k}^{i} \bar{B}_{k} u_{k}+L_{k}^{i}\left(y_{k}-\bar{C}_{k}^{i} \hat{\bar{x}}_{k}^{i}\right)+N_{k}^{i} y_{k+1}, \\
r_{k}^{i}=y_{k}-\bar{C}_{k}^{i} \hat{\bar{x}}_{k}^{i}
\end{array},\right.
$$

$$
i=1, \cdots, M
$$

where $\hat{\bar{x}}_{k}^{i}$ denotes the augmented state estimation of the $i$ th filter, and $r_{k}^{i}$ denotes the residual vector of the $i$ th filter. Matrices $T_{k}^{i}, N_{k}^{i}$ and $L_{k}^{i}$ are designed according to the filter design method proposed in Section 2.

Similar as the methodology in [18], the residual $r_{k}^{i}$ can be considered as a quality indicator of the $i$ th sensor fault estimation filter. In other words, $r_{k}^{i}$ will be close to zero if the $i$ th faulty model is accurate. Otherwise, $r_{k}^{i}$ will deviate from zero. Given a proper threshold $\epsilon$, we present the following fault detection scheme.

Fault detection scheme: If all $\left\|r_{k}^{i}\right\| \leq \epsilon, i=$ $1, \cdots, M$, then there is no fault. Otherwise, if any $\left\|r_{k}^{i}\right\|>$ $\epsilon, i=1, \cdots, M$, a fault is detected

As mentioned before, if the $i$ th faulty model is accurate, then $r_{k}^{i}$ will be close to zero while $r_{k}^{j}, j \neq i$ will deviate from zero. Therefore, the residual corresponding to the actual faulty model exhibits the minimum norm. Based on this observation, the following fault isolation scheme is proposed. by

Fault isolation scheme: The fault mode is estimated

$$
\hat{F}_{k}=F_{k}^{i^{*}}
$$

where $i^{*}$ is the fault mode index corresponding to the residual with minimum norm, i.e.

$$
i^{*}=\underset{i}{\arg } \min _{i=1, \cdots, M}\left\{\left\|r_{k}^{i}\right\|\right\}
$$

Once the fault mode is estimated, the fault magnitude $f_{k}$ can be estimated as follows

$$
\hat{f}_{k}=\left[\begin{array}{ll}
0 & I_{q}
\end{array}\right] \hat{\bar{x}}_{k}^{i^{*}}
$$

It is concluded that the sensor faults can be detected, isolated by the proposed fault diagnosis strategy and then be estimated by equation (36).

\section{Simulations}

In this section, a numerical example is used to illustrate the effectiveness of the proposed method.

Example 1. Consider the discrete-time system in the form of (1) with the following parameters

$$
\begin{gathered}
A_{k}=\left[\begin{array}{ccc}
0.2 e^{-k / 100} & 0.6 & 0 \\
0 & 0.5 & \sin (k) \\
0 & 0 & 0.7
\end{array}\right], B_{k}=\left[\begin{array}{l}
1.3 \\
0.5 \\
0.6
\end{array}\right] \\
D_{k}=\left[\begin{array}{lll}
1 & 0 & 0 \\
0 & 1 & 0 \\
0 & 0 & 1
\end{array}\right], C_{k}=\left[\begin{array}{lll}
1 & 0 & 0 \\
0 & 1 & 0
\end{array}\right]
\end{gathered}
$$

and the fault distribution matrix $F_{k}$ belongs to the following set

$$
F_{k} \in \mathcal{F}_{k} \triangleq\left\{F_{k}^{1}, F_{k}^{2}\right\}
$$

where

$$
F_{k}^{1}=\left[\begin{array}{l}
1 \\
0
\end{array}\right], F_{k}^{2}=\left[\begin{array}{l}
0 \\
1
\end{array}\right]
$$

In the simulation, the control input is $u_{k}=2 \sin (0.05 k)$, the initial state is $x_{0}=\left[\begin{array}{lll}0.4 & -0.7 & 0.2\end{array}\right]^{T}$, and the covariance matrices of the process noise and measurement noise sequences are $Q=0.05^{2} I_{3}, R=0.05^{2} I_{2}$.

In this situation, matrices $E, \bar{A}_{k}, \bar{B}_{k}, \bar{D}_{k}, \bar{C}_{k}^{1}, \bar{C}_{k}^{2}$ are determined by (32). A solution to equation (8) is obtained by simply choosing the matrix $S$ in (13) and (14) as

$$
S=\left[\begin{array}{lllllll}
1 & 0 & 0 & 0 & 0 & 0 & 0 \\
0 & 1 & 0 & 0 & 0 & 0 & 0 \\
0 & 0 & 1 & 0 & 0 & 0 & 0 \\
0 & 0 & 0 & 1 & 0 & 0 & 0 \\
0 & 0 & 0 & 0 & 1 & 0 & 0
\end{array}\right]
$$

Then, by using equations (13), (14), (18) and (19), the gain matrices $T_{k}^{1}, T_{k}^{2}, N_{k}^{1}, N_{k}^{2}, L_{k}^{1}, L_{k}^{2}$ and the variance matrices $P_{k}^{1}, P_{k}^{1}$ can be recursively determined.

To illustrate the performance of the proposed method, the following sensor fault is considered

$$
F_{k}=F_{k}^{2}, f_{k}=\left\{\begin{array}{cc}
0 & k<50 \\
1.2 & k \geq 50
\end{array}\right.
$$

In this case, $\left\|r_{k}^{1}\right\|$ and $\left\|r_{k}^{2}\right\|$ are depicted on Fig. 2. It is shown in Fig. 2 that $\left\|r_{k}^{1}\right\|$ exceeds the threshold. As a sequence, a sensor fault is detected. It should also be noticed that $\left\|r_{k}^{2}\right\|$ is close to zero despite the occurance of fault. In other words, $\left\|r_{k}^{2}\right\|$ is insensitive to this fault. Therefore, it can be concluded that the fault mode is $F_{k}^{2}$, i.e. the second sensor is faulty.
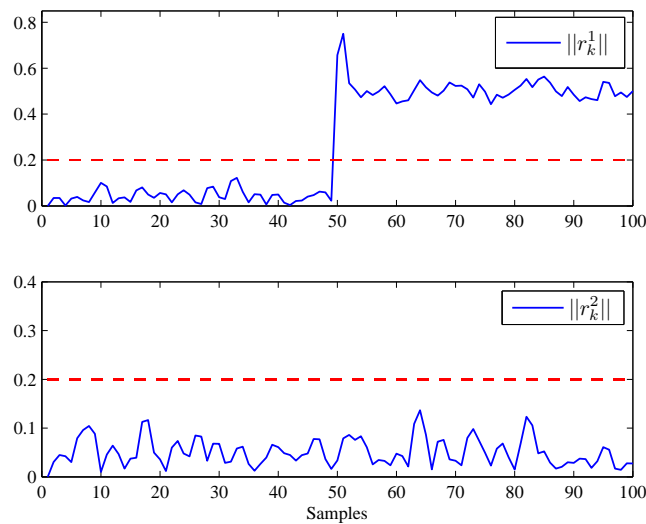

Figure 2 The residuals of sensor fault estimation filters in an abrupt fault scenario

Note that the fault mode is $F_{k}^{2}$, then the fault estimation provided by the 2ed filter is accurate. The fault estimation result is depicted in Fig. 3, where the actual fault is illustrated by dashed lines and the estimation is represented by solid lines. From Fig. 3, it can be seen that the abrup$\mathrm{t}$ fault can be accurately estimated by the proposed fault estimation filter.

Remark 2. It is noted that the fault estimate previous to 50th sample is zero. The reason is that the fault estimate should be set as zero until a fault occurance is detected. 


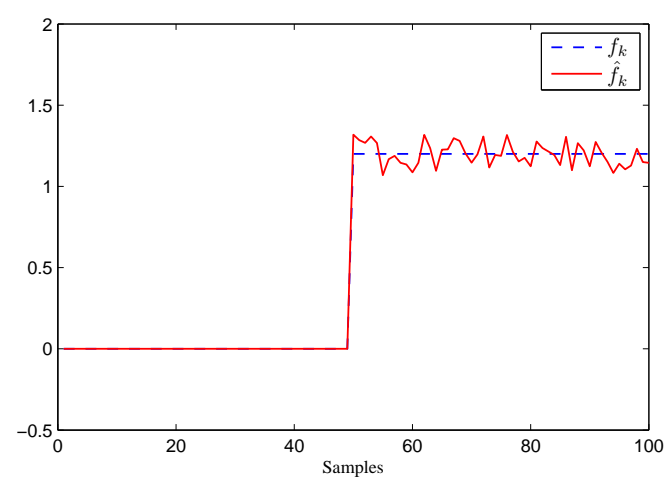

Figure 3 The fault estimation result in an abrupt fault scenario

In order to demonstrate the ability of the proposed method in dealing with time-varying faults, the following fault is simulated

$$
F_{k}=F_{k}^{1}, f_{k}=\left\{\begin{array}{cc}
0 & k<30 \\
\sin (0.2 k-6) & k \geq 30
\end{array}\right.
$$

In this situation, $\left\|r_{k}^{1}\right\|$ and $\left\|r_{k}^{2}\right\|$ are depicted on Fig. 4. It is seen that $\left\|r_{k}^{1}\right\|$ is close to zero but $\left\|r_{k}^{1}\right\|$ exceeds the threshold. This implies that the first sensor is faulty. In addition, the fault estimation result is depicted in Fig. 5. From Fig. 5, it can be seen that the fault estimation starts from the 32th sample, which is the fault detection time. This means that the fault detection time-delay is only 2 samples, even if there is a time-varying fault occurred. Moreover, Fig. 5 also illustrates that the proposed fault estimation filter exhibits satisfactory performance in estimating time-varying fault.
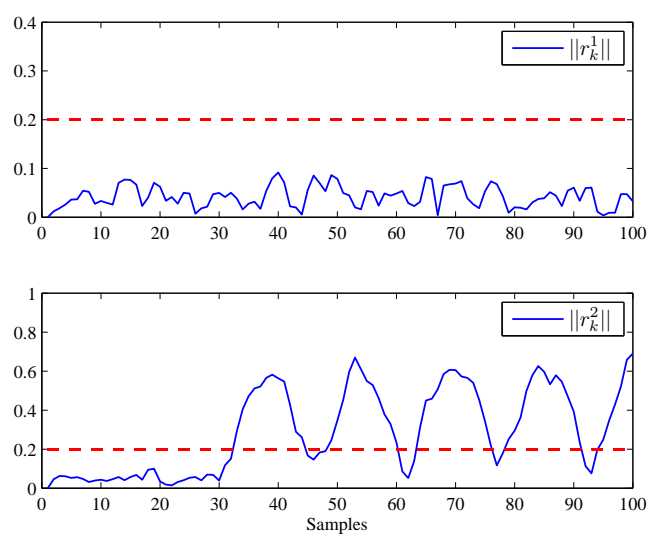

Figure 4 The residuals of sensor fault estimation filters in a timevarying fault scenario

\section{Conclusion}

This paper proposes a novel sensor fault diagnosis approach for discrete-time LTV systems using descriptor system technique. The main advantage of the presented method lies in its ability to detect, isolate and estimate sensor faults in the presence of process noise and measurement noise. Simulation results show the effectiveness of the proposed method.

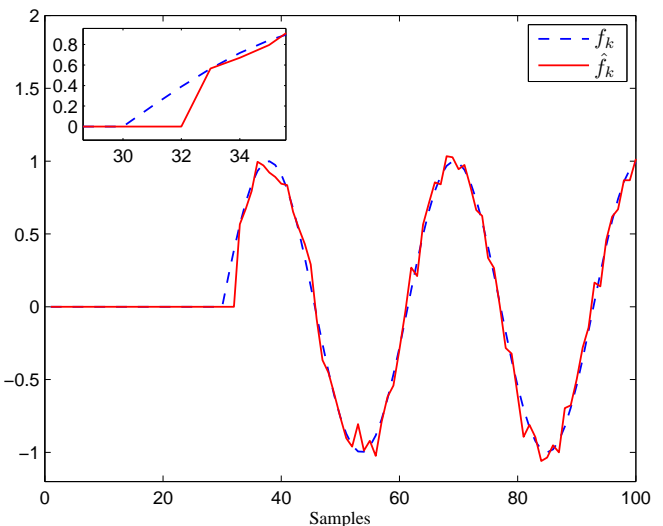

Figure 5 The fault estimation result in a time-varying fault scenario

In addition, it should be mentioned that the descriptor system approach merit further research. One of the possible future directions is to extend the results developed in this paper to networked control systems ${ }^{[22,23]}$ or systems with stochastic hybrid dynamics ${ }^{[24-26]}$.

\section{Acknowledgement}

The authors would like to acknowledge the anonymous reviewers for their helpful comments and suggestions which improve the presentation of this paper.

\section{References}

1 Chen J, Patton R. Robust Model-Based Fault Diagnosis for Dynamic Systems. Boston: Kluwer Academic Publishers, 1999

2 Blanke M, Kinnaert M, Lunze J, Staroswiecki M. Diagnosis and Fault-Tolerant Control. Berlin: Springer-Verlag, 2006.

3 Ding S X. Model-Based Fault Diagnosis Techniques. Berlin: Springer, 2008.

4 Frank P M. Fault diagnosis in dynamic systems using analytical and knowledge based redundancy - A survey and some new results, Automatica, 1990, 26(3): 459-474.

5 Dai X, Gao Z. From model, signal to knowledge: A data-driven perspective of fault detection and diagnosis, IEEE Transactions on Industrial Informatics, 2013, 9(4): 2226-2238.

6 Wang H, Daley S. Actuator fault diagnosis: an adaptive observer-based technique, IEEE Transactions on Automatic Control, 1996, 41(7): 1073-1078.

7 Jiang B, Chowdhury F. Observer-based fault diagnosis for a class of nonlinear systems, In: Proceeding of the 2004 American Control Conference, Boston, USA: IEEE, 2004. $5671-5675$.

8 Zhang K, Jiang B, Cocquempot V. Adaptive observer-based fast fault estimation, International Journal of Control, $\mathrm{Au}$ tomation, and Systems, 2008, 6(3): 320-326.

9 Demetriou M A, Polycarpou M M. Incipient fault diagnosis of dynamical systems using online approximators, IEEE Transactions on Automatic Control, 1998, 43(11): $1612-1617$.

10 Hamdi H, Rodrigues M, Mechmeche C, Benhadj Braiek N. Robust fault detection and estimation for descriptor systems based on multi-models concept, International Journal of Control, Automation, and Systems, 2012, 10(6): 1260-1266.

11 Hamdi H, Rodrigues M, Mechmeche C, Theilliol D, Benhadj B N. Fault detection and isolation in linear parametervarying descriptor systems via proportional integral observer, International Journal of Adaptive Control and Signal Processing, 2012, 26(3): 224-240. 
12 Jiang B, Chowdhury F N. Fault estimation and accommodation for linear MIMO discrete-time systems, IEEE Transactions on Control Systems Technology, 2005, 13(3): 493-499.

13 Zhang K, Jiang B, Shi P. Observer-based integrated robust fault estimation and accommodation design for discretetime systems, International Journal of Control, 2010, 83(6): $1167-1181$.

14 Astorga-Zaragoza C M, Theilliol D, Ponsart J C, Rodrigues M. Fault diagnosis for a class of descriptor linear parametervarying systems, International Journal of Adaptive Control and Signal Processing, 2012, 26(3): 208-223.

15 Zhong M-Y, Liu S, Zhao H-H. Krein space-based $\mathrm{H}_{\infty}$ fault estimation for linear discrete time-varying systems, Acta $A u-$ tomatica Sinica, 2008, 34(12): 1529-1533.

16 Gao Z, Ho D W C. State/noise estimator for descriptor systems with application to sensor fault diagnosis, IEEE Transactions on Signal Processing, 2006, 54(4): 1316-1326.

17 Gao Z, Wang H. Descriptor observer approaches for multivariable systems with measurement noises and application in fault detection and diagnosis, Systems \& Control Letters, 2006, 55(4): 304-313.

18 Rodrigues M, Sahnoun M, Theilliol D, Ponsartc J-C. Sensor fault detection and isolation filter for polytopic LPV systems: A winding machine application, Journal of Process Control, 2013, 23(6): 805-816.

19 Wang Z, Shen Y, Zhang X. Attitude sensor fault diagnosis based on Kalman filter of the discrete-time descriptor system, Journal of Systems Engineering and Electronics, 2012, 23(6): 914-920.

20 Wang Z, Shen Y, Zhang X, Wang Q. Observer design for discrete-time descriptor systems: an LMI approach, Systems \& Control Letters, 2012, 61(6): 683-687.

21 Ben-Israel A, Greville T. Generalized Inverses: Theory and Applications (2ed). New York: Springer, 2003.

22 Zhang L, Gao H, Kaynak O. Network-induced constraints in networked control system $C$ A survey, IEEE Transactions on Industrial Informatics, 2013, 9(1): 403-416.

23 You K-Y, Xie L-H. Survey of recent progress in networked control systems, Acta Automatica Sinica, 2013, 39(2): $101-117$.

24 Zhang L. $H_{\infty}$ estimation for piecewise homogeneous Markov jump linear systems, Automatica, 2009, 45(11): 2570-2576.

25 Zhang L, Lam J. Necessary and sufficient conditions for analysis and synthesis of Markov jump linear systems with incomplete transition descriptions, IEEE Transactions on $\mathrm{Au}$ tomatic Control, 2010, 55(7): 1695-1701.

26 Li Y-Y, Zhong M-Y. On optimal fault detection for discretetime Markovian jump linear systems, Acta Automatica Sinica, 2013, 39(6): 926-932.

WANG Zhen-Hua Assistant Professor with the Department of Control Science and Engineering, Harbin Institute of Technology. He received his Ph. D. degree from the Department of Control Science and Engineering, Harbin Institute of Technology in 2013. His research interests include fault diagnosis, faulttolerant control and observer design.

E-mail: zhwang1987@gmail.com

RODRIGUES Mickael Associate Professor in Claude Bernard University of Lyon 1 with the Automatic and Process Control Laboratory (LAGEP), France. He received his Ph. D. degree in Automatic Control from the Department of Automatic Control of the Henri Poincaré University of Nancy 1, in 2005 from the Centre de Recherche en Automatique de Nancy (CRAN). His current research interests are focused on modelbased fault diagnosis, fault tolerant control, multi-models, LPV systems, singular/descriptor systems, observers, stability and LMI.

E-mail: mickael.rodrigues@univ-lyon1.fr

THEILLIOL Didier Full Professor in Research Centre for Automatic Control of Nancy (CRAN) at University of Lorraine. $\mathrm{He}$ received his $\mathrm{Ph}$. D. degree in Control Engineering from University of Lorraine (France) in 1993. His current research interests include sensor and actuator model-based fault diagnosis
(FDI) method synthesis and active fault-tolerant control (FTC) system design for LTI, LPV, Multi-linear systems and also reliability analysis of components.

E-mail: didier.theilliol@univ-lorraine.fr

SHEN Yi Professor with the Department of Control Science and Engineering, Harbin Institute of Technology. He received his Ph. D. degree from the Department of Control Science and Engineering, Harbin Institute of Technology in 1995. His current research interests include fault diagnosis, flight vehicle control and ultrasound signal processing. Corresponding author of this paper.

E-mail: shen@hit.edu.cn 